\title{
Innovative approach to higher education management as an important factor of sustainable economic development
}

\author{
Irina Zhukovskaya ${ }^{1}$, Svetlana Begicheva ${ }^{2, *}$, and Dmitry Nazarov ${ }^{2}$ \\ ${ }^{1}$ Tashkent State Economic University, I. KarimovAv., 49, 100003 Tashkent, Uzbekistan \\ ${ }^{2}$ Ural StateUniversity of Economics, 8MarchStr., 62, 620144 Ekaterinburg, Russia
}

\begin{abstract}
The article shows that innovative approaches to the organization of management of higher education institutions are an important factor in the sustainable development of the economy. In particular, this paper proposes a model for optimizing managerial decisions based on the conducted causal analysis of socio-economic systems. Structural equation modeling (SEM) is widely used to verify conceptual models that determine the direction and strength of causal relationships. The paper proposes to combine the SEM results with the analysis carried out by methods of fuzzy cognitive modeling. This synthesis of models supports what-if analysis and makes it possible to determine the optimal control strategy. The implementation of the proposed model was carried out on data set from the survey of students of the Tashkent State University of Economics studying remotely through a digital platform at the Ural State University of Economics. The purpose of the survey was to analyze student loyalty. The calculations were performed taking into account various input configurations corresponding to specific control strategies
\end{abstract}

\section{Introduction}

In the context of the formation of the digital economy, the introduction of innovative technologies into the activities of enterprises and organizations has become not just a means of optimizing the internal processes of economic objects, but an urgent need in a highly competitive environment. It is the introduction of innovative technologies that provides new opportunities for any organization to speed up work, allows you to stay ahead of competitors when making operational or strategic decisions, helps to optimize management processes and achieve competitive advantages in the economic market.

In the modern period, such a concept as sustainable education is gaining importance. To achieve the sustainability of higher education, it is necessary to develop methodological foundations, technological mechanisms and optimize the functioning of all available resources. Competent management decision-making in the context of sustainable economic

* Corresponding author: begichevas@mail.ru 
development is unthinkable without the use of economic and mathematical models, tools and methods of advanced information and communication technologies.

In this regard, in this paper, the authors present the results of a comprehensive study carried out in higher educational institutions of the Russian Federation and Uzbekistan to develop a methodology and develop optimal solutions for the effective organization of management in a higher educational institution in order to achieve the release of competitive specialists for industries and sectors of the economy.

\section{Methodology of Modeling and Decision-making}

The goal of the methodology is to build a modeling environment taking into account causeand-effect relationships when building a fuzzy cognitive map and consists of the following stages:

1) Structural modelling: building a model that estimates SEM-validated causal relationships [1].

2) Fuzzy cognitive modelling: formalization of a fuzzy cognitive map in the form of an adjacency matrix, including indicators of the power of influence between factors obtained as a result of SEM $[2,3]$.

3) Modeling of control scenarios: operation of the model and carrying out calculations with sets of values of input factors, which, in our case, define control strategies [4,5].

4) Analysis of modeling results, selection of the optimal solution: determination of the optimal configuration of input factors that maximizes the target.

\section{Implementation of the Model}

\subsection{Structural Modelling}

The respondents to this study were undergraduates of the Tashkent State University of Economics studying remotely through a digital platform at the Institute of Distance Education of the Ural State University of Economics during the 2020 pandemic. Data collection was carried out within one month. A total of 425 people took part in the survey.

The questionnaire developed for the study was based on questionnaires from the works of A. Dehghan [6] and O.Helgesen [7], which were adapted and finalized with the participation of experts: specialists in educational and methodological work and information technologies of the Institute of Distance Education. The survey questions were divided into six constructs $[8,9]$. Here is a description of each construct.

Service (Perceived Quality of Learning). The construct questions assessed the educational and methodological support of the learning process, as well as the communicative interaction of teachers with students;

Technology (The perceived quality of the technologies used). The questions of this construct were related to the technical and software support of the educational process;

Trust (Trust in the staff of the university). The construct issues concerned the degree to which the student is convinced that the university staff always act in his best interests and keep their promises;

Satisfaction (Student Satisfaction). Assessment of the perceived difference between the perceived and real image of the product [10]; 
Reputation (Academic reputation of the university). The degree to which the student is convinced that university staff are always acting in their best interests or fulfilling their promises;

Loyalty (Student loyalty). The intention of students to cooperate with the university, the willingness to give recommendations to enter the university to familiar applicants.

The respondents were asked to give a rating for each of the 15 questions of the questionnaire. Answers to the questions were scored on a five-point Likert scale from $1=$ strongly disagree to $5=$ strongly agree.

To construct a graphical representation of the model - a path diagram (Figure 1), the software for modeling with structural equations Smart PLS was used.

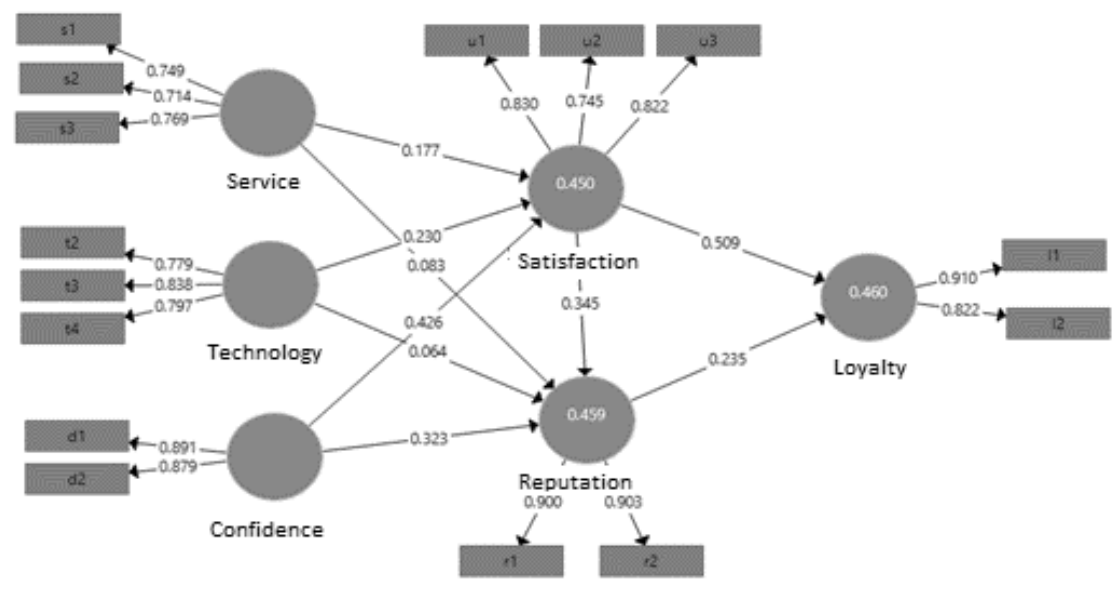

Fig.1. Structural model

For several iterations, satisfactory values of the correspondence indicators of the theoretical and empirical matrices were achieved, demonstrating the consistency of the model $(\chi 2=12.65, p=0.21$, GFI $=0.99$, RMSEA $=0.05)$. The estimates of the correlation coefficients between the model variables are statistically significant $(\mathrm{p}<0.05)$.

\subsection{Fuzzy Cognitive Modelling}

The values of the correlation coefficients obtained during the construction of the structural model, reflecting the strength of the connection between the factors, are used to compile the adjacency matrix.

$$
E=\begin{array}{cccccc}
0 & 0 & 0 & 0.177 & 0.083 & 0 \\
0 & 0 & 0 & 0.230 & 0.064 & 0 \\
0 & 0 & 0 & 0.426 & 0.323 & 0 \\
0 & 0 & 0 & 0 & 0.345 & 0.509 \\
0 & 0 & 0 & 0 & 0 & 0.235 \\
0 & 0 & 0 & 0 & 0 & 0
\end{array}
$$




\subsection{Simulation of Control Scenarios}

Operation of the model and carrying out calculations with sets of values of input factors is performed using the adjacency matrix $E$, which is a fuzzy cognitive matrix of interactions. The simulation procedure is carried out by multiplying the input vector representing the state of each input factor that sets the control strategy by a fuzzy cognitive matrix of mutual influences. The values of the input vector elements can be equal to 1 (the maximum value of the factor impact) or 0 (there is no factor impact).

Seven possible sets of input data for three factors are shown in Table 2. The calculation is carried out in three iterative stages, taking into account all levels of connections of the fuzzy cognitive map.

Consider the calculation procedure using the example of the input vector

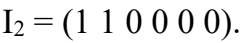

At the first iteration, we multiply the vector $\mathrm{I}_{2}$ by the adjacency matrix $\mathrm{E}$ and obtain the values of the output vector $\mathrm{O}_{1}$ :

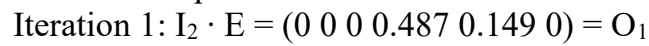

The input vector for the second iteration is the sum of the output vector after iteration 1 and the input vector of iteration 1 . When performing the multiplication in iteration 2 , the following result is obtained:

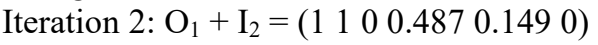

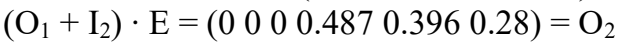

To complete the loop of iterations, you need to perform the third iteration:

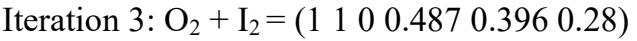

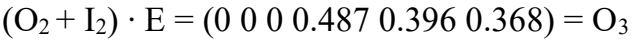

The calculation results for each input configuration are shown in Table 1. The change in output parameters is indicated in percent. The rows of the table are sorted in descending order of data in the "Loyalty" column.

Table 1. Input data and results of iterations

\begin{tabular}{|c|c|c|c|c|c|c|}
\hline \multirow{2}{*}{ Opt. } & \multicolumn{3}{|c|}{ Input data } & \multicolumn{3}{c|}{ Result (3 iterations) } \\
\cline { 2 - 7 } & Service & Technology & Confidence & Satisfaction & Reputation & Loyalty \\
\hline 3 & 1 & 1 & 1 & $90 \%$ & $81 \%$ & $71 \%$ \\
\hline 7 & 0 & 1 & 1 & $82 \%$ & $67 \%$ & $62 \%$ \\
\hline 4 & 1 & 0 & 1 & $49 \%$ & $56 \%$ & $43 \%$ \\
\hline 2 & 1 & 1 & 0 & $49 \%$ & $40 \%$ & $37 \%$ \\
\hline 6 & 0 & 0 & 1 & $41 \%$ & $42 \%$ & $34 \%$ \\
\hline 5 & 0 & 1 & 0 & $41 \%$ & $26 \%$ & $28 \%$ \\
\hline 1 & 1 & 0 & 0 & $8 \%$ & $14 \%$ & $9 \%$ \\
\hline
\end{tabular}

\subsection{Selecting the Best Solution}

The optimal configuration of input factors that maximizes the target loyalty indicator corresponds to a set of 3 values of input indicators. Figure 2 shows a histogram of the values of the output indicators corresponding to the sets of values of each input factor of the model. The set number is located on the vertical axis of the histogram. The horizontal axis reflects the value of the resulting indicators. Results are listed in descending order, starting with the maximum value corresponding to row 3 . 


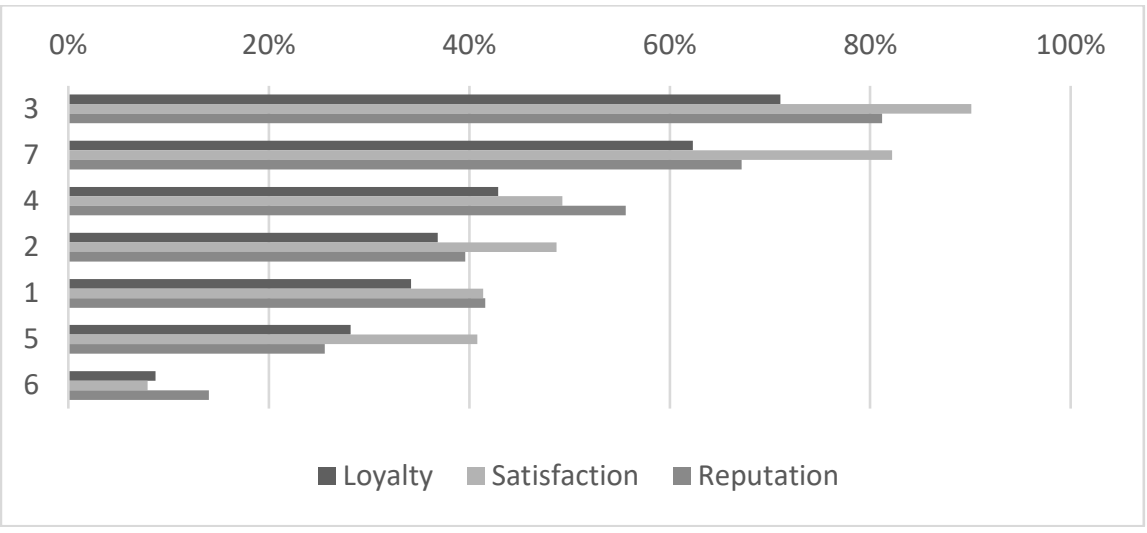

Fig. 2. Histogram of the resulting indicators

As mentioned above, the optimal solution is achieved in option 3 when all input factors are activated. The result is justified since all factors have a positive impact. Although the optimal solution is identified in case 3, the strength of the influence of each input factor on the result is different. This can be seen by analyzing options 1, 5, 6. By activating the influence of the Service factor, a result of $9 \%$ was obtained. Acting on loyalty through the Technology factor, a value of $28 \%$ was obtained. When using the Trust factor, the loyalty level will rise by $34 \%$. These considerations are helpful in choosing investment strategies for a university. At the same time, it should be taken into account that it is not easy to set a strategy based on the Trust factor, but, as the analysis of the model emphasizes, this factor influences student loyalty more than others, since its action has the greatest effect. On the contrary, the factors Technology and Service are more easily embedded in the definition of the management strategy, since they are under the direct control of the university [4-6]. For example, the head of a university can help improve the service and quality of education by investing in the impact on the human factor, in infrastructure, in cooperation with other academic institutions, etc.

\section{Conclusions and Recommendations}

The article presents a model of optimization of management decisions for strategic planning based on fuzzy cognitive maps built using the results of structural modeling.

Fuzzy cognitive maps make it possible to represent in the form of a causal graph the causal relationships that arise during the development of a certain economic object, but the assessment of the weights expressing the degree of causality between the two concepts of the model can cause difficulties even for highly qualified and experienced experts. The article proposes a method for assessing the weights of relationships by analyzing survey data using structural modeling methods. The set of weights of the edges of the causal graph determined using SEM is written as a square adjacency matrix of the cognitive map. Further, by multiplying the vectors of the input states of the factors by the adjacency matrix, a simulation experiment is carried out. The best strategy is determined by the set of input parameters, the use of which gives the maximum value of the resulting indicator. The advantage of the proposed model can be attributed to the fact that simultaneously with the allocation of such a set of strategic actions, the decision maker gets the opportunity to analyze and compare the effects for all alternative scenarios. This allows you to make the best decision based on the real capabilities of the organization.

The approach described in the article can have a fairly wide application in research based on survey data and aimed at studying consumer or employee behavior. The 
integration of modeling methods with structural equations and fuzzy cognitive modeling allows taking into account the impact of implicit factors, which are a measure of the subjective perception of the respondents, on the objective indicators of the organization's performance. We see the development of research results in the further improvement of the technique of using fuzzy cognitive maps to analyze the influence of qualitative factors, often subject to subjective assessment, on an objectively measured resultant indicator.

\section{References}

1. A. Aktepe, S. Ersz, B Toklu, Computers \& Industrial Engineering, 86 (2015)

2. S. Lee, H. Ahn, Expert Systems with Applications, 36 (2009)

3. A.V. Zagranovskaya, Yu.N. Eissner, Modern economics: problems and solutions, 10 (2017)

4. B. Kosko, Prentice-Hall International (1991)

5. D.M. Nazarov, D.A. Azarov, S.V. Begicheva, Application of Information and Communication Technologies (2016)

6. A. Dehghan, Political Science (2012)

7. O. Helgesen, E. Nesset, Corporate Reputation Review, 10 (2007)

8. T. Henning-Thurau, M.F. Langer, U. Hansen, Journal of Service Research, 3 (2001)

9. A.R. Rodie, S.S. Kleine, Handbook of Services Marketing and Management, 2 (2002)

10. I. Zhukovskaya, Faculty of Management Bulletin UNECON, 4 (2018) 